\title{
Distinctions genrées et identités discursives dans la généricité du discours d'investiture: le cas de Kirchner, Rousseff et Sirleaf
}

\author{
Dorgelès Houessou \\ Professeur à l'Université Alassane Ouattara, Bouaké, Côte d'Ivoire. \\ dorgeleshouessou@yahoo.fr
}

Résumé: L'arrière plan théorique de cette communication est l'analyse du discours. Le but visé est d'interroger sur un double plan méthodologique et notionnel les rapports entre les modalités formelles des distinctions genrées en sciences du langage et le genre spécifique du discours d'investiture en tant que discours politique de légitimation. Montrer les procédés de mise en œuvre discursive de la masculinité et de la féminité comme posture revient, dans un tel contexte de passation/réception du pouvoir politique, à relever les mécanismes par lesquels des identités génériques projettent un parcours ethotique en fonction des attentes sociologiques (mythes et stéréotypes) liées aux schèmes identitaires concernés. Il en est ainsi parce que les conditions de la construction de l'image de l'orateur résident dans la perception doxique préexistante au discours et simultanément dans l'acte de langage en tant que composante subjective élémentaire de celui-ci.

Mots clés: Genre. Femme et pouvoir. Discours d'investiture. Ethos de féminité. Argument de genre.

Resumo: O contexto teórico deste artigo é a análise do discurso. O objetivo é interrogar, tanto a nível metodológico quanto conceitual, a relação entre as modalidades formais de distinções de gênero nas ciências da linguagem e o gênero específico do discurso da investidura enquanto discurso político de legitimação. Mostrar os processos de implementação discursiva da masculinidade e da feminilidade como postura nos leva a encarar, nesse contexto de passagem / recebimento do poder político, os mecanismos pelos quais as identidades genéricas projetam um percurso etótico de acordo com as expectativas sociológicas (mitos e estereótipos) relacionados com os esquemas identitários em causa. Isso ocorre porque as condições para a construção da imagem do orador residem na percepção dóxica preexistente ao discurso e, simultaneamente, no ato da fala como um componente subjetivo elementar próprio.

Palavras-chave: Gênero. Mulher e poder. Discurso de investidura. Ethos de feminilidade. Argumento de gênero. 
Introduction: Considérations générales sur le discours d'investiture (DI)

Nos recherches sur la catégorisation du discours d'investiture 1 nous ont conduit à formuler quelques postulats définitoires du genre concerné, à savoir qu'il s'agit d'un discours politique, d'un discours rituel et d'un discours épidictique. En tant que discours politique, le DI constitue une parfaite illustration de la nécessité de l'instance politique de communiquer à l'instance citoyenne le bien-fondé de ses intentions et de ses actions (CHARAUDEAU, 2005, p. 16). De même comme discours rituel, il vise à pérenniser un système politique et l'allégeance des citoyens audit système. Les DI présidentiels servent ainsi, par l'entremise du rituel où ils prennent place, dans une magie incantatoire propre au performatif, à amener à la réalité l'objet de leur énonciation du fait même de leur seule énonciation. À en croire Charaudeau, "Le couple contemplation/action pose la question de la force créatrice du langage. Simples accumulations d'évidences constatives que les présidents assènent, les discours d'investiture sont souvent des performatifs masqués" (CHARAUDEAU, 2005, p. 47). Ils laissent constater notamment que le chef de l'exécutif accepte ses nouvelles fonctions et entend améliorer l'existence de ses citoyens. "Ceci dit, ces allocutions forment bien un groupe: elles sont toutes prononcées par des présidents qui cherchent à profiter de leur prise de fonction pour marquer une rupture dans un sens progressiste avec le passé" (CHARAUDEAU, 2005, p. 93).

Comme discours épidictique c'est-à-dire construit sur une propension au jugement mélioratif du sujet investi et de ses alliés, le DI présidentiel a pour rôle de légitimer le sujet politique dans sa nouvelle fonction, car "une fois le serment prononcé, le nouveau président se retrouve dans la position de l'énonciateur légitime par excellence [...] le discours fonctionne comme un

\footnotetext{
${ }^{1}$ Désormais DI. Cf.: Pour une approche conceptuelle du discours politique: contribution à la généricité du discours d'investiture. Cas de la Côte d'Ivoire de 2000 à 2011, Thèse soutenue publiquement le 03 décembre 2013 à l'Université Félix Houphouet Boigny d'Abidjan-Cocody. Je reprends ici en vrac quelques postulats développés dans ma thèse. Je renvoie aussi sur la question à trois articles à savoir: "Notes sur la généricité du discours d'investiture", Revue du Ltml N 9 , Octobre 2013; "Retórica da imagem e temática da união no discurso icônico em torno da tomada de posse de Alassane Ouattara". Tradução: Isabel Cristina Michelan de Azevedo. Revisão da tradução: Eduardo Lopes Piris. EID\&A - Revista Eletrônica de Estudos Integrados em Discurso e Argumentação, Ilhéus, No 5, p. 56-73, juil/dez. 2013 et "La performance générique comme condition de dénomination du genre du discours d'investiture", dans Question de genre ou genre en question, approches interdisciplinaire de la généricité, Selim Yilmaz (éds.), Actes du colloque de Mascara les 16/17 Décembre 2014, Munich, Lincom GmbH, 2015, pp. 92-114.
} 
langage autorisé" (CHARAUDEAU, 2005, p. 149). Cette dimension du DI présidentiel est assez importante pour en constituer une intention communicative définitoire. Elle est ainsi associée à la nature ostentatoire du rituel d'investiture présidentiel servant de contexte au DI et destinée à révéler au public cible le caractère inviolable de la souveraineté et de l'autorité du président investi. Elle est aussi le lieu de célébration des valeurs communes aux membres du groupe où l'investiture se déploie de même que des valeurs personnelles, propres à l'orateur et à son public. Il s'agit donc d'un discours fondateur car s'il fonde dans une perspective macrostructurale l'Etat en sa déclinaison idéologique, s’il définit les choix dont le principe est organisationnel pour une société donnée, il réinvente par la même occasion l'Etat considéré dans une optique de renouvellement de ses valeurs. Cette mécanique en fait un discours paradoxal à plusieurs titres (BENOIT À LA GUILLAUME, 2000).

De fait, lorsque le sujet investi se présente et est présenté par l'autorité chargée de l'investir comme l'émanation d'un vœu commun à tous les membres du groupe au sein duquel l'investiture s'actualise, le DI se fait célébration à la fois d'un système politique dont il marque la continuité et d'une conception collective de ce qui doit constituer l'idéal social envisagé par tous. Cette faculté à instaurer un ordre politique, donc à fonder de nouveau une société tendue vers un idéal de société, est fédératrice.

II demeure toutefois que le DI, sous une telle ambition de rassemblement et d'osmose générale est un discours d'exclusion. II n'est construit que sous l'ambition de l'intégration pour mieux rappeler à une catégorie du groupe concerné son échec quant à l'appropriation du pouvoir, car il n'y est question que de pouvoir. Dans une telle perspective, il se veut expiatoire du vice de la contestation en ce qu'il fonde la légitimité du sujet investi. L'objet d'une investiture étant prioritairement de rappeler de manière ostentatoire qu'un individu est détenteur du pouvoir ou de l'autorité qui était jusque là vacante et que par la magie sociale des rites associés à cette ostentation - dont la parade dans le cas de l'investiture présidentielle l'investiture rend incontestable ledit pouvoir et ladite autorité. Le DI construit ainsi nécessairement une dynamique de communion et d'exclusion. D'où la thématique de la souveraineté et les appels constants au rassemblement qui lui sont consubstantiels. Ces deux modalités font du DI un discours de 
manipulation comme tout performatif car "la performativité repose sur une " mise en scène de la légitimité”, sur la production d'une autorité crédible, et en ce sens se dissémine à l'infini en des transformations qui se conforment aux normes de la crédibilité sans jamais parfaitement coïncider avec elles" (OGER, 2006, p. 128).

Le corpus analysé dans cette étude, faute d'espace dans le cadre d'un article, comprend des fragments des DI de trois présidentes. Il s'agit de Mmes Cristina Fernández De Kirchner, Dilma Rousseff et Ellen Johnson Sirleaf, toutes trois ayant été respectivement présidentes de l'Argentine, du Brésil et du Libéria, des pays qui ont en commun non seulement un passé colonial mais encore une perception doxique du genre féminin non encore émancipée des stéréotypes les plus réducteurs en dépit des différences géographiques et linguistiques. De fait, il s'agit de discours prononcés lors de leur première accession au pouvoir et qui ont d'historique leur caractère sans précédent quant à l'investiture de femmes présidentes². Ces discours, prononcés au moment de leur accession à la plus haute magistrature nationale, sont dignes d'intérêt pour le sujet qui nous occupe. Il s'agit donc de déterminer dans leurs productions, leur regard non seulement sur leur genre, mais aussi et surtout sur la tentative de réappropriation des valeurs doxiquement attachées aux femmes par le genre dominant en vue de la mise en évidence de leur compétence et de leur légitimité car "c'est en effet dans la rupture entre le signe et ses usages, entre le performatif et son contexte que git la promesse de "resignification subversive" qui peut permettre aux victimes des discours de haine de les "vider de leur charge d'humiliation" et de se réapproprier leur force, au profit d'un "discours insurrectionnel” (OGER, 2006, p. 129). De ce point de vue, les postures de ces locutrices jouent aussi bien sur les stéréotypes liés au genre que sur la contestation de ceux-ci.

En considérant que le genre est une construction discursive, sa performativité dans un DI féminin lève la question de la posture discursive. Comme le note Jami reprenant Butler "Si les identités de genre sont

\footnotetext{
${ }^{2}$ Exception faite de l'Argentine qui a connu une présidente antérieure en la personne de María Estela Martínez Cartas dite Isabel Martínez de Perón ou Isabel Perón. En tant que vice-présidente, élue en 1973 comme colistière de son mari Juan Domingo Perón, elle lui succède comme présidente après la mort de celui-ci en 1974. Elle dirige l'Argentine du 1er juillet 1974 au 24 mars 1976 avant d'être déposée par un coup d'État pour raison d'illégitimité entre autres. Sa non contemporanéité et le mode d'accession au pouvoir ne relevant pas du suffrage direct sont les raisons de l'absence de son discours d'investiture dans notre corpus.
} 
construites et constituées par le langage, cela signifie qu'il n'y a pas d'identités de genre qui précèdent le langage. Ce sont le langage et le discours qui 'font' le genre. Les actes de genre ne sont pas 'performés' par le sujet, mais ils constituent de façon performative un sujet qui est l'effet, plutôt que la cause, du discours" (JAMI, 2008, p. 214). Cette posture justifie peut-être le silence des chancelières et femmes d'État européennes sur la revendication d'une identité féminine dans leur DI, contrairement à celles dont les discours font l'objet de la présente étude. Ainsi, ne point affirmer sa féminité en situation d'investiture apparait, dans des sociétés plus émancipées quant aux stéréotypes sexistes, comme la résultante d'une subversion visant à tenir sous le boisseau les préjugés misogynes en faisant fi même de leur existence. Cet argument du silence dont on sait depuis Perelman qu'il permet non seulement "d'éviter une décision relative à une incompatibilité" (2008, p. 267), mais aussi de construire des "palliatifs aux dangers de l'ampleur" (2008, p. 643), permet surtout aux dirigeantes européennes, qui ne mentionnent aucunement leur genre dans leurs DI, de manifester ainsi un ethos de féministe sans prêter le flanc à une polémique sur la question même. La seule revendication performative de l'acte de réception de l'investiture dans leur contexte serait fondée sur le sexe (évidence physiologique) et non sur le genre (construction sociale).

\section{I- Identités genrées dans le DI}

\section{I-1 Généralités}

Le point de départ de cette étude est la théorie de l'argumentation de Ruth Amossy selon laquelle l'argumentation consiste fondamentalement dans “les moyens verbaux qu'une instance de locution met en œuvre pour agir sur ses allocutaires en tentant de les faire adhérer à une thèse, de modifier ou de renforcer les représentations et les opinions qu'elle leur prête, ou simplement d'orienter leur réflexion sur un problème donné" (AMOSSY, 2000, p. 27.). À partir d'un tel point de vue, il semble justifié de tenir pour acquise la prise en compte de la doxa comme construction discursive qui influence en amont l'énonciateur. Non pas qu'il tienne l'impensé doxique pour vrai ou juste mais en cela qu'il lui incombe de le prendre en considération dans la représentation qu'il se fait de son auditoire en vue d'orienter les réflexions de celui-ci. D'où la 
négociation identitaire opérée durant toute argumentation entre un "je" locuteur et un "tu" allocutaire, tous deux stratifiés en postures qui sont autant d'identités énonciatives en interaction autour d'un compromis dialectique.

Le concept d'identité tel que nous l'envisageons donc ici à la suite de Lamizet est indissociable de celui de légitimité. Au sujet de la légitimité en tant que contrainte du discours politique, Charaudeau fait remarquer que celle-ci est constitutive de l'identité du personnage politique. De ce point de vue, pour qu'un discours soit reconnu comme politique, il est primordial que le sujet qui l'assume soit autorisé à le dire, soit en vertu d'un rôle institutionnel, soit en raison de son charisme qui lui donne le privilège d'être écouté; c'est le cas par exemple de leaders d'opinion qui n'exercent aucune autorité institutionnelle. Lamizet note en ces termes la prépondérance de l'identité dans tout processus de légitimation:

[...] ce qui fonde la légitimité d'un pouvoir, d'une pratique politique ou d'un engagement, c'est la conformité de cette pratique ou de l'exercice de ces pouvoirs avec les logiques qui structurent l'identité des acteurs qui les exercent ou celle des acteurs sur qui ils sont exercés (LAMIZET, 2002, p. 188).

Un processus d'identification est en effet nécessaire pour lequel les administrés ou la communauté qu'il représente, reconnaissent à l'auteur d'un discours politique, la légitimité de ses propos. Une telle légitimité justifie d'ailleurs non seulement le discours de l'autorité concernée mais aussi ses faits et gestes car tout en elle est indice sémiotique et doit conforter la représentativité dont elle se réclame précisément.

Le cas du DI présidentiel est l'un des exemples les plus achevés de cet amalgame car à la question "qui parle ?", le public répond invariablement "le président (ou la présidente) de la République”; en d'autres termes, l'identité de statut du locuteur du DI prime sur l'identité de rôle au point que cette dernière se confond à la première. S'il en est ainsi, c'est parce que l'investiture est au sens propre l'endossement d'une identité symbolique ${ }^{3}$, à la fois celle de l'État que le sujet investit incarne, et celle de tous les sujets dont ce dernier est fait porte parole. Cette alchimie identitaire à relent de légitimation est perçue

\footnotetext{
3 L'étymologie du terme "investiture" renvoie d'ailleurs à la mise du vêtement et à la remise d'accessoires liés à un titre.
} 
différemment selon que le président investi soit un homme ou une femme. Ceci parce que des habitus bien établis font du pouvoir un objet masculin et de la féminité un statut péjoratif comparé à la masculinité. Derville et Pionchon évoquent même " la prégnance de représentations sociales qui sont largement antagonistes avec l'engagement politique au féminin ", car dans la plupart des sociétés humaines la tendance est à l'obédience patriarcale (DERVILLE; PIONCHON, 2008, p. 53). ${ }^{4}$

Dans ce contexte d'hostilité comment la performativité du DI féminin est-elle perçue? Le rapport des femmes au langage a fait l'objet de nombreuses études. Aebischer (1985, p. 15) notamment distingue quatre types de femmes: traditionnelle, nouvelle, moderne et suffragette. Elle estime que si les femmes traditionnelles et nouvelles admettent que le bavardage est un trait distinctif du féminin, il reste un fait péjoratif. Quant aux femmes modernes et suffragettes, elles se réapproprient le bavardage comme l'expression valorisante d'une “identité spécifiquement féminine”. Ferencuhova note aussi qu'il existe une dualité de l'image et de la parole qui distingue l'homme de la femme de sorte que la parole de l'un et l'autre soit conforme à l'image doxique qui lui préexiste. Elle déclare ainsi:

\begin{abstract}
Dans une perspective du monde occidental patriarcal, on pourrait dire, grosso modo, que la femme a été depuis longtemps condamnée à être montrée (en tant que l'idéal de la beauté, éloquente par sa visibilité et pourtant muette), tandis que l'homme s'est approprié le pouvoir de signifier, donc d'avoir le droit à la parole, d'être le porteur du discours dominant, de créer un univers spirituel, par opposition à la femme qui est en quelque sorte le symbole de la matérialité (FERENCUHOVA, 2003, p. 3).
\end{abstract}

La représentation de la femme, envisagée sous cette œillère à vocation dégradante, est la plus répandue à travers le monde si l'on considère, au nombre des causes probables de cet imaginaire, les sacro-saints principes des cultes monothéistes identifiant l'être suprême comme étant de genre masculin, et les femmes comme des tentatrices destinées à maintenir l'humanité dans les méandres de la bassesse dont la matérialité est

\footnotetext{
${ }^{4}$ Sur ce point d'ailleurs les concepts de "hiérarchie entre les sexes", "valence différentielle des sexes", et de "domination masculine" respectivement développés par DUMONT (1979), HERITIER (1996) et BOURDIEU (1998), montrent l'universalité de la discrimination sexiste dont les femmes sont l'objet et qui les disqualifient d'un point de vue doxique dans les rôles sociaux majeurs dont celui de l'exercice du pouvoir.
} 
l'expression. Une telle vision des femmes s'enrichit même d'un dédoublement herméneutique dont Ferencuhova fait cas en ces termes:

Or ce schéma peut fonctionner aussi sous une forme inverse, celle des stéréotypes de la vie banale où la femme est considérée comme un être bavard, gaspillant ses paroles, les semant partout et créant verbalement un univers virtuel, fictif, furtif, éphémère et donc vain... L'homme par contre est défini comme un être de l'action, de la création solide et de ce qui est solide (tant au niveau matériel que spirituel) (FERENCUHOVA, 2003, p. 3).

Ainsi, une double désacralisation doxique du genre féminin voudrait dans un premier temps que ce dernier n'ait pas droit à la parole donc aux instruments légitimes de l'expression du pouvoir, et dans un second temps que cette parole, si elle lui échoit comme par le plus grand des hasards, ne soit guère fondée, ni rationnellement, ni à propos, dans la mesure où il lui serait préférable l'utilité de l'action doxiquement définie comme étant d'obédience masculine. D'où la tendance de certaines présidentes investies à revendiquer leur féminité comme par défi au sexisme et à en développer une rhétorique particulière dont les hommes seraient incapables dans le même contexte. Cette forme de rhétorique genrée vise en effet pour ces dernières à réaffirmer leur appartenance au genre féminin. Ce qui est impossible aux hommes qui passeraient sinon pour des rustres misogynes et machistes et surtout pour la raison que le pouvoir est doxiquement masculin et qu'un homme au pouvoir relèverait, stéréotypiquement parlant, de la norme. ${ }^{5}$ Si licence il y a dans ce cas de figure, elle concerne le discours politique de campagne visant à disqualifier le genre féminin comme ce fut le cas entre Sarkozy et Segolène Royal en 2007 (OLIVESI, 2007). Mais si le discours de campagne autorise une telle réappropriation doxique des préjugés liés au genre féminin, et pourrait conduire comme ce fut le cas de Sarkozy, un candidat à mettre en avant son tropisme genré masculin, ce procédé deviendrait contre-productif dans un DI. À preuve Sarkozy n'a point fait explicitement référence à sa masculinité durant son investiture quoiqu'il ait à maintes reprises traité son adversaire d'incompétente durant la campagne et en jouant sur le dogme de l'incompétence politique féminine dont parle Oger en affirmant que:

\footnotetext{
${ }^{5}$ Sur ce point les réflexions de Coulomb-Gully (2013) sont d'un grand intérêt. Elle montre en effet, dans la perspective d'une perception doxique de la masculinité du pouvoir, comment le discours de campagne de Nicolas Sarkozy a implicitement révélé une masculinité forte contre une tentative de féminisation de François Hollande affublé d'une masculinité molle.
} 
"l'argument de l'incompétence fonctionne comme un opérateur central du passage de l'argumentation polémique à la disqualification injurieuse" (OGER, 2009, p. 30). Le DI est donc un genre exceptionnel en la matière car si la doxa ne permet pas aux hommes d'y promettre qu'ils dirigeront comme des hommes - la référence excessive aux figures de la puissance et de la force induisant un risque majeur d'interprétation en faveur de l'autocratisme - cela est en revanche possible chez les femmes.

\section{I-2 L'argument ad hominem contre l'argument ad mulierem}

En distinguant l'argument ad hominem de l'argument ad personam, Perelman conclut que le premier se rapporte au cadre du préjugé, à la cohérence ou à l'incohérence des superstitions et croyances d'un auditoire restreint - par opposition à l'auditoire universel - alors que le second se définit comme " une attaque contre la personne de l'adversaire et qui vise à le discréditer " (PERELMAN; OLBRECHTS-TYTECA, 2008, p. 150). L'argument ad mulierem désigne pour sa part la forme spécifiquement sexiste de l'argument ad hominem.

En proie au discours misogyne de la société, la tendance féministe, en général, concevra comme déterminant de se réapproprier l'identité féminine et de présenter comme valorisantes toutes les valeurs négatives ou péjoratives stéréotypiquement attribuées au "sexe faible" par les hommes. Ainsi, par glissement métonymique, toute revendication de l'identité féminine opérée par une femme revient implicitement à porter un jugement accusateur sur les hommes à l'origine de la dévalorisation des femmes. Un tel procédé est de mise dans les DI de notre corpus comme le montrent les extraits suivants:

Chers Brésiliens et chères Brésiliennes,

De par la décision souveraine du peuple, l'écharpe présidentielle sera portée aujourd'hui par une femme pour la première fois.

C'est avec ce courage que je vais gouverner le Brésil.

Mais les femmes, ce n'est pas seulement du courage. C'est de la tendresse aussi. De la tendresse que je consacre à ma fille et à mon petit fils.

De la tendresse pour serrer ma mère dans mes bras, elle m'accompagne et me bénit. 
C'est avec cette immense tendresse que je veux m'occuper de mon peuple et lui consacrer les prochaines années de ma vie. ${ }^{6}$

(Dilma Rousseff)

Le premier paragraphe du premier extrait constitue l'introduction de l'adresse de Dilma Rousseff. Celle-ci définit d'entrée de jeu son tropisme genré et l'exhibe comme un signe de fierté personnelle et nationale. Cette précision sur son genre pourrait sembler fortuite mais il n'en est rien, car il s'agit d'un pied de nez au sexisme dont les relents sont multiculturels et pesant dans la société latino-américaine. Affirmer sa féminité et la fierté qui en découle est donc une preuve de l'émancipation des femmes qui montrent ainsi que les carcans doxiques destinés à les dégrader sont dépassés.

Le second paragraphe du premier extrait traduit une ambivalence de valeur féminine. Dilma Rousseff se définit ainsi non seulement comme étant courageuse mais aussi et surtout comme une femme tendre. Elle associe la tendresse au genre féminin et revendique une telle valeur comme fondamentalement féminine. Elle réussit donc par le truchement de l'argument ad hominem, formulé contre le sexisme, à revendiquer sa féminité et à poser comme évident le syllogisme explicite selon lequel toutes les femmes sont tendres et courageuses, or Dilma Rousseff est une femme, donc Dilma Rousseff est tendre et courageuse. Ce syllogisme explicite est amplifié par la triple occurrence anaphorique du syntagme: "de la tendresse". Ainsi, en plus d'affirmer sa féminité comme valeur utile à la bonne gouvernance, la présidente investie martèle comme un slogan cette réappropriation du préjugé doxique selon lequel la tendresse est une valeur féminine, qui est inopérante voire préjudiciable dans le domaine politique. Elle en fait par resémantisation une valeur cardinale au succès politique, et gage de sa disponibilité à en user sur le plan national. Pour ce faire elle établit un parallèle métaphorique entre sa famille et sa nation. De la première découle la tendresse filiale et maternelle que la présidente se propose d'instituer en modèle de gouvernance par transfert métonymique. Car son exemple de succès établi trouve sa source dans la présence et la bénédiction maternelle dont elle dit avoir toujours bénéficié. Ainsi lorsqu'elle se propose d'être une mère pour son peuple, c'est bien dans l'optique de garantir à ce dernier les

\footnotetext{
${ }^{6}$ Discours d'investiture de la présidente Dilma Rousseff, $1{ }^{\text {er }}$ janvier 2011, traduction disponible en ligne: www.voltairenet.org/article168186.html
} 
mêmes dispositions aux succès et à l'épanouissement. Une posture favorable donc au stéréotype de la femme mère-poule comme le montre la clausule de ce passage. Elle est en effet révélatrice de cet épidictique mélioratif du féminin puisque l'argument par la volonté d'agir autant que l'emploi du verbe "consacrer" qui connote le sacrifice personnel et évoque l'isotopie du religieux, constituent des indices qui s'enrichissent de la figure ethotique de la mère protectrice.

Ce deuxième extrait est de nature thématique similaire car il porte sur l'acceptation voire la revendication d'une valeur revendiquée comme personnelle voire féminine: la sincérité ou l'honnêteté.

\begin{abstract}
Je sais qu'il manque beaucoup de choses, je sais que nous devrons en corriger d'autres. Je suis convaincue que nous serons en mesure de le faire avec l'effort et le travail de tous les Argentins. Aussi - parce vous savez que la sincérité est l'une de mes valeurs cardinales - je sais qu'il m'en coûtera parce que je suis une femme, parce que vous pouvez toujours être une travailleuse, vous pouvez être une professionnelle ou une femme d'affaires, mais votre féminité vous coûtera toujours plus cher. J'en suis absolument convaincue. ${ }^{\text {? }}$
\end{abstract}

(Cristina Fernández De Kirchner)

Quoique Cristina Kirchner se dise consciente de la difficulté à être une femme en vertu de la faiblesse doxiquement associée au "sexe faible", elle pose comme unique porte de sortie à cette fatalité doxique la qualité qu'elle dit définitoire de sa personnalité, à savoir la sincérité. Ainsi, en posant comme une évidence que la société est machiste et fait payer sans répit aux femmes la moindre erreur, qu'elles soient "ouvrières, professionnelles ou chefs d'entreprises", la présidente investie se réapproprie le regard de la société sur sa condition féminine et lui renvoie son mépris en y répondant par la force de "l'honnêteté". Au terme d'une resémantisation implicite, un procédé argumentatif par généralisation sourd de ce passage. Ainsi, de personnelle, la sincérité (qui n'est pas doxiquement une valeur féminine en raison du mythe de la femme tentatrice et fatale) devient une qualité genrée propre à toutes les femmes.

\footnotetext{
${ }^{7}$ Discurso de la Presidenta de la Nacion, Cristina Fernández De Kirchner, en el acto de asunción del mando en el congreso de la nacion ante la asamblea legislativa 10/12/2007, Presidencia de la Nación Argentina [en ligne]. Disponible sur: http://www.presidencia.gob.ar/discursos/2940. Nous traduisons.
} 
Dilma Rousseff fait de l'argument du genre un usage des plus abondants. Dans l'extrait suivant elle rend hommage à la femme et célèbre toutes les femmes brésiliennes pour leur féminité:

Je sais également combien la douceur de la soie jaune et verte de l'écharpe présidentielle n'est qu'apparente, car elle est porteuse d'une énorme responsabilité par-devant la nation.

Afin de l'assumer pleinement, je porte en moi la force et l'exemple de la femme brésilienne. Mon cœur est ouvert pour recevoir en ce moment une étincelle de son immense énergie. Et je sais que mon mandat devra refléter avec générosité cette audace du scrutin populaire qui, après avoir élu à la présidence un ouvrier, décide de convoquer une femme pour diriger le destin du pays.

Je viens pour ouvrir des portes afin que de nombreuses femmes puissent également à l'avenir devenir présidentes; et pour que - en ce jour - toutes les Brésiliennes ressentent de la fierté et de la joie d'être femmes.

Je ne viens pas pour vanter les mérites de mon histoire personnelle, mais pour rendre hommage à la vie de chaque femme brésilienne. Mon engagement primordial, je le répète, est de rendre honneur aux femmes, de protéger les plus faibles et de gouverner tous les Brésiliens ! ${ }^{8}$

"Force", "immense énergie" et "audace" sont donc des valeurs partagées par toutes les femmes brésiliennes et implicitement par celles du monde entier selon Dilma Rousseff. Il est important de noter "la force de l'exemple" à laquelle elle souhaite faire accéder sa victoire qui de personnelle est présentée comme une victoire du genre féminin. En prétendant ouvrir la voie de la présidence à de "nombreuses femmes", la présidente investie est convaincue que son sacre est vécu comme une source de "joie" et de "fierté" par toutes les femmes. Il s'agit ici d'une généralisation typiquement féminine en raison de la solidarité générique dont les femmes peuvent faire preuve de manière explicite dans un tel contexte à la différence des hommes, comme une sorte de revanche sur le sexe coupable de les avoir longtemps privées de la parole institutionnelle. En affirmant que son "engagement primordial est de rendre honneur aux femmes", elle tient à relever le défi de la compétence dont son genre est privé et dit incapable par le sexisme. Elle inscrit dès lors son mandat dans une logique de justification du genre féminin comme si elle en était un emblème à l'aune duquel toutes les femmes seraient jugées coupables de ses échecs ou artisanes de ses victoires.

\footnotetext{
${ }^{8}$ Nous soulignons.
} 


\section{I-2 L'argument par l'autorité du genre}

Il s'agit ici, pour une femme, de la convocation d'une personne du même genre en vue de montrer le caractère universel de la vérité et de la justesse de l'émancipation féminine. Ainsi dans les extraits suivants les présidentes investies en appellent à la parenté qui les lie à des femmes politiques en vue d'opérer un transfert métonymique mélioratif de valeurs et d'identité politique.

Mes chers libériens: Aujourd'hui, en vous parlant, je tiens à dire que je suis très heureuse de l'importance des délégations des gouvernements étrangers et de nos partenaires internationaux et locaux qui sont venus se joindre à nous pour célébrer ce triomphe de la démocratie dans notre pays. Je suis particulièrement émue par la présence des Femmes Parlementaires de l'Union Africaine et d'autres de mes sœurs, qui participent aujourd'hui avec nous à la solidarité. Permettez-moi de mentionner en particulier la présence de Son Excellence Mme Laura Bush, épouse du Président des États-Unis d'Amérique, Son Excellence Condoleezza Rice, Secrétaire d'État des États-Unis, et d'autres membres de la délégation américaine. Mme Bush et moi partageons une passion et un engagement communs en faveur de l'égalité des sexes et de l'éducation des filles. Je la salue pour son travail en Afrique et dans la région du golfe Persique. Je la remercie également ainsi que le secrétaire d'État Rice pour leur présence et leur soutien. ${ }^{9}$

(Ellen Johnson Sirleaf)

Je me suis rappelé alors une très belle photo parue il y a quelques jours de notre chère compagne, amie et présidente de la République Fédérale du Brésil, Dilma Rousseff, très jeune aussi, quand elle a été emprisonnée et j'ai pensé un instant qu'aujourd'hui Dilma occupe la présidence d'un des pays les plus importants du monde. Peut-être que cette jeune femme ${ }^{10}$ aurait pu être assise là à la place que j'occupe. ${ }^{11}$

(Cristina Fernández De Kirchner)

La présidente libérienne en associant à son investiture l'image de l'Union Africaine des Femmes Parlementaires, de Laura Bush, épouse du Président américain et de Condoleezza Rice, Secrétaire d'État des États-Unis

\footnotetext{
9 Discours inaugural de H. E. Ellen Johnson Sirleaf, le 16 janvier 2006, en ligne, disponible sur http://www.emansion.gov.Ir/doc/inaugural_add_1.pdf. Nous traduisons et soulignons.

${ }^{10}$ La jeune fille dont il est question se nomme Ana Teresa Diego (1954-1976). Elle était une étudiante d'astronomie de l'Université de La Plata, détenue disparue pendant la dernière dictature civico-militaire argentine. Par décision de L'Union astronomique internationale, l'astéroïde 11441 porte aujourd'hui son nom.

${ }^{11}$ Discours de la Présidente de la Nation, Cristina Fernández de Kirchner, dans l’acte de prendre le commandement du Congrès National devant l'Assemblée législative, le 10/12/2011, Présidence de la Nation Argentine [en ligne]. Disponible sur: http://www.presidencia.gob.ar/discursos/25601-actode-asuncion-de-mando-en-elcongreso-de-la-nacion-discurso-de-la-presidenta-de-la-nacion. Nous traduisons et soulignons.
} 
d'Amérique, exprime sa gratitude quant à leur solidarité. Ce comportatif sert toutefois des intérêts plus grands car il s'agit par transfert métonymique de revendiquer la compétence des tierces citées dont l'aura et la dimension internationales ne font plus aucun doute. Le rapport entre l'énonciatrice et l'épouse du président américain par exemple est dissymétrique car la présidente investie en révélant la passion commune de l'équité entre les genres et de l'éducation de la jeune fille qui les unit toutes deux, salue les résultats engrangés par Laura Bush dans la lutte pour les questions liées au genre et semble promettre implicitement de lui emboiter le pas même si cette dernière n'a pas la charge effective de l'exécutif aux États-Unis. L'argument du genre prévaut cependant dans l'insistance sur la qualification le leur féminisme comme étant aussi bien une "passion" (élan pulsionnel) qu'un "engagement" (choix rationnel). Ce rapport dissymétrique entre elle et les autres femmes citées, qui ne bénéficient pas du même droit statutaire que l'énonciatrice, est aussi minoré par l'emploi du lexème "sœurs" employé par la présidente investie pour revendiquer, plus qu'une solidarité de fait, une sororité de droit entre les femmes de pouvoir présentes à son investiture.

Le même rapport est établi par Cristina Kirchner avec Dilma Rousseff dont elle évoque l'amitié et l'histoire douloureuse, l'emprisonnement alors qu'elle était très jeune et le militantisme brimé par un régime autocratique. Le rapport en question est toutefois symétrique car elle finit au bout de cet épisode pathique à évoquer la place qui est la sienne au moment de l'énonciation, c'est-à-dire comme lieu d'expression du pouvoir ultime et de prééminence statutaire (este mismo lugar en donde estoy sentadayo). Car l'objet de ce double ancrage narratif est de montrer d'une part, les douloureux travers de la dictature par le biais de l'histoire de la jeune Ana Teresa Diego, et de la jeune future présidente Dilma Rousseff, et d'autre part, que son histoire en tant que présidente investie aurait pu être celle de n'importe quelle autre femme qui aurait eu le courage qu'ont manifesté Ana Teresa Diego et Dilma Rousseff en s'opposant à l'autoritarisme. Ce passage est encore un appel à la dignité et à la droiture que chaque femme devrait, à en croire l'énonciatrice, incarner pour laisser son nom à la postérité. 


\title{
II- Parcours ethotique du genre dans le DI et références mythologiques
}

\author{
Pour Amossy:
}

L'ethos est l'image que l'orateur construit de lui-même dans son discours afin de se rendre crédible. Fondé sur ce qu'il montre de sa personne à travers les modalités de son énonciation, il doit assurer l'efficacité de sa parole et sa capacité à emporter l'adhésion du public. Dans ce cadre, l'ethos fait partie d'une entreprise de persuasion délibérée dans laquelle il est mobilisé au même titre que le logos et le pathos. Fruit d'un savoir-faire, il renvoie nécessairement à un sujet intentionnel qui programme sa présentation de soi en fonction de ses objectifs propres (AMOSSY, 2010, p. 25).

Il s'agit, dans cette définition, de l'ethos discursif c'est-à-dire l'image que l'orateur construit de lui-même pendant son discours. En tant que dit, posé et implicite, l'ethos discursif se différencie de l'ethos prédiscursif qui relève du capital de sympathie ou d'antipathie qu'un sujet locutif nourrit dans l'opinion avant sa prise de parole. L'ethos préalable ou prédiscursif est donc “ l'ensemble des données dont on dispose sur le locuteur au moment de sa présentation de soi “(AMOSSY, 2010, p. 25). Charaudeau résume ainsi cet état de fait:

[...] le sujet apparait au regard de l'autre dans une identité psychologique et sociale qui lui est attribuée, et en même temps se montre à travers l'identité discursive qu'il se construit. Le sens que véhiculent nos paroles dépend à la fois de ce que nous sommes et de ce que nous disons. L'ethos est le résultat de cette double identité, mais qui finit par se fondre en une seule (CHARAUDEAU, 2005, p. 89).

Ainsi, par un subtil effet de complémentarité, si l'ethos prédiscursif crée un horizon d'attente concernant le discours d'un tiers et les orientations supposées que celui-ci pourrait prendre, il revient à l'ethos discursif de confirmer ou d'infirmer lesdites orientations. Cette renommée dans le cadre des distinctions genrées est négative pour la femme en général en vertu de stéréotypes pesants.

Le DI offre l'occasion de consolider les valeurs communes au groupe au sein duquel l'investiture a lieu. Ces valeurs se résument pour certaines leaders à mettre en avant la sensibilité doxiquement liée à leur genre et relevant du mythe de la mère nourricière. ${ }^{12}$ II s'agit des ethos d'identification définis par

\footnotetext{
${ }^{12}$ Sur ce point Badinter (2010) et Hrdy Blaffer (2002) lèvent le voile sur le caractère de sensibilité ou d'empathie que les femmes manifesteraient naturellement du fait de leur "rôle social de
} 
Charaudeau. Ce dernier conçoit en effet l'identification comme le processus par lequel un citoyen, membre d'une communauté, laisse son identité être aliénée par celle de l'homme politique en tant qu'il incarne une identité collective ou toutes les particularités identitaires se fondent. Ce processus passe par une implication discursive dans l'affect social par la construction d'imaginaires capables de fédérer un très grand nombre de citoyens. Cette raison pousse, selon Charaudeau, les hommes politiques en quête de légitimité, dans l'espoir qu'une grande majorité de citoyens se reconnaitront en eux, à revendiquer des valeurs parfois contradictoires comme les couples: traditionalisme / modernisme, sincérité / ruse, puissance / modestie, élitisme / populisme etc. L'identification passe par la construction des ethos de "puissance", de "caractère", d"'intelligence", d"'humanité", de "chef" et de "solidarité". Nous nous intéresserons ici aux ethos de solidarité et d'humanité manifestés dans notre corpus.

\section{II-1- L'ethos d" “humanité"}

Il est utile pour l'homme politique de montrer qu'il peut avoir du cœur et se sentir proche de ses concitoyens dans les épreuves qu'ils traversent. Cette faculté, c'est précisément l'ethos d'“humanité”. Comme le fait remarquer Charaudeau:

L'ethos d' "humanité" est également un imaginaire important pour l'image de l'homme politique. "Etre humain" se mesure à la capacité de faire preuve de sentiment, de compassion envers ceux qui souffrent, mais c'est aussi savoir avouer ses faiblesses, montrer quels sont ses goûts, jusqu'aux plus intimes: Pour être un homme politique, on n'en est pas moins homme (CHARAUDEAU, 2005, p. 114).

Cette remarque sous-entend que l'homme politique est doxiquement perçu comme un surhomme, un homme qui ne s'encombre pas de sentiments et qui n'a pas de faiblesse. Car les faiblesses que pourrait avouer un homme politique sont de l'ordre de la banalité. Autrement il en irait du suicide politique. II reste que si les hommes doivent afficher un tel ethos pour un certain public, le sentiment ou l'émotion de même que la sensibilité sont des faiblesses stéréotypiquement accolées aux femmes quand la société fait de

reproduction". Les deux auteures montrent que le concept de "mère nourricière" vient d'un stéréotype conçu par le discours dominant des hommes sous la houlette de récits mythiques. 
l'homme un être ferme, posé et placide. ${ }^{13} \mathrm{Si}$ "faire preuve de sentiment" est doxiquement jugé comme allant de soi chez les femmes, c'est parce que la sensibilité, l'émotivité et la compassion sont partie intégrante des traits de caractères entrant dans le stéréotype féminin. On pourrait en conclure que dans les représentations sociales du genre, l'ethos d'humanité serait un ethos féminin c'est-à-dire "naturellement" exprimé par les femmes. Cela dit, la propension des présidentes dont nous analysons les DI à en faire un usage "naturel" ne peut paraitre autrement que comme une actualisation assumée de leur féminité envers de tels préjugés réducteurs. Les extraits ${ }^{14}$ suivants en sont l'illustration (nous soulignons):

Je n'aurais de cesse tant qu'il y aura des Brésiliens sans nourriture, des ménages vivant dans la désolation des rues, des enfants pauvres abandonnés à leur sort. L'harmonisation des familles se fait dans le partage des aliments, dans une ambiance de paix et de joie. C'est ce rêve que je poursuis !

Je répète ce que j'ai dit pendant la campagne électorale, je préfère le bruit que fait la presse libre au silence de la dictature. Ceux qui, comme moi et tant d'autres de ma génération, ont lutté contre l'arbitraire et la censure et la dictature, sommes tombés naturellement amoureux de la plus complète démocratie et de la défense intransigeante des droits de l'homme dans notre pays et en tant que bannière sacrée de tous les peuples.

Je voulais vous dire que j'ai consacré toute ma vie à la cause du Brésil. J'ai dédié ma jeunesse, comme beaucoup d'entre vous ici présents, au rêve d'un pays juste et démocratique.

(Dilma Rousseff)

Nous ferons sourire à nouveau les enfants. Ces milliers d'enfants incapables d'avoir une carte d'électeur mais qui m'ont répété chaque fois que je les rencontrais et leur serrais la main qu'ils ont voté pour moi. En effet, ils ont voté avec leurs cœurs. À ces enfants et à tous les autres enfants libériens à travers le pays, je vous dis, je vous aime très très fort. Je travaillerai dès aujourd'hui pour vous donner de l'espoir et un avenir meilleur.

(Ellen Johnson Sirleaf)

\footnotetext{
${ }^{13}$ Voir notamment à ce sujet les développements de ROCHEBLAVE-SPENLE (1964).

${ }^{14}$ Nous soulignons.
} 
Dilma Rousseff se définit d'emblée comme une femme sensible à la misère de ses populations et, par le biais d'une parole de promesse, entend persuader l'auditoire de sa détermination à lutter contre l'indigence tant qu'elle subsistera. Cette figure du sentiment dont elle fait montre par compassion évolue en commisération car elle revendique son histoire personnelle, les souffrances et injustices endurées par elle, comme gage de l'évidence de son combat. L'implicite ainsi distillé est que son investiture ne représente pas une fin, mais tout juste une "réalisation" susceptible de lui fournir les moyens de continuer son combat contre l'injustice et la précarité. La figure du sentiment transparait aussi dans le rêve qu'elle dit nourrir et enfin dans l'expression "tomber naturellement amoureux". Si le rêve est opposé à l'action et que cette dialectique révèle stéréotypiquement une discrimination genrée, alors Dilma Rousseff revendique ainsi son féminisme de même qu'à travers sa "déclaration d'amour".

Cette figure du sentiment se manifeste aussi à travers celle de la mère nourricière également campée par Ellen Johnson Sirleaf qui propose un témoignage émouvant de sa rencontre avec des enfants. Sous l'ambition métaphorique de redonner le sourire aux enfants, la présidente investie promet de mettre tout en œuvre pour que la classe la plus faible de la société - en l'occurrence celle des enfants - soit protégée et heureuse. La figure du sentiment est double dans un tel passage car l'épidictique en faveur des enfants dont elle loue l'innocence, car ils n'ont pu voter et sont pourtant victimes des travers de la société, se révèle en définitive comme une exhortation à reconnaitre son caractère maternel et sa sensibilité, bref son humanité. On retrouve également chez elle cette "déclaration d'amour" quasi maternalisée amplifiée par la réduplication du marqueur intensif "très très fort" et dont l'opinion doxique ne s'accommoderait qu'avec difficulté si elle était faite par un homme.

Dans l'extrait ${ }^{15}$ suivant Christina Kirchner en fait de même.

Aujourd'hui, je ne veux pas partager des chiffres ou des données avec vous ni vous parler des choses que nous avons faites au cours des quatre ans et demi qui ont été si importants, ${ }^{16}$ la renégociation [de la dette], le paiement du Fonds

\footnotetext{
${ }^{15}$ Nous soulignons.

${ }^{16}$ La présidente n'évoque pas, comme pourrait le croire tout lecteur non averti, le bilan de son époux qui est le président sortant, mais son propre bilan en tant que sénatrice fédérale. Un signe
} 
[de solidarité], la lutte acharnée contre le le chômage, l'indigence, la pauvreté dans laquelle nous gagnons des batailles et des triomphes importants, et non le définitif, car la victoire définitive manquera toujours tant qu'il y aura un pauvre dans la patrie. Cela est très clair.

(Cristina Fernández De Kirchner)

La prétérition dont fait usage Christina Kirchner est en soi un procédé rhétorique à valeur ethotique car elle laisse entrevoir ici l'image d'une présidente dont l'humilité ne saurait souffrir qu'elle se vante d'un bilan élogieux. D'où le déni de l'obsession des chiffres que le circuit énonciatif impose de traduire en faits concrets dont l'énumération retranscrit bien l'ampleur et l'importance. Son inclination à lutter contre la pauvreté de manière systématique donne d'elle ici l'image d'une présidente compatissante qui au lieu de s'enorgueillir de ses résultats qu'elle reconnait “importants", se propose de combattre sans répit la misère dans son pays.

Ces extraits quoique brefs témoignent de la revendication d'un ethos féministe présenté comme nécessaire à la bonne gouvernance. Si Dilma Rousseff évoque son histoire personnelle empreinte de persécution par un régime totalitaire, Ellen Johnson Sirleaf évoque sa relation particulière avec les enfants tout comme Christina Kirchner pour qui les pauvres méritent toute l'attention au point d'occulter un bilan pourtant très important. Ces passages sont révélateurs de l'intérêt pour ces femmes de camper des figures traditionnellement perçues comme féminines sans risquer d'exagérer ou d'incommoder l'auditoire.

\section{II-2- L'ethos de solidarité}

Charaudeau définit ainsi l'ethos de solidarité:

L'ethos de "solidarité" fait de l'homme politique un être qui non seulement est attentif aux besoins des autres mais les partage et s'en rend comptable. La solidarité se caractérise par la volonté d'être ensemble, de ne pas se distinguer des autres membres du groupe et surtout de faire corps avec eux dès l'instant que ceux-ci se trouvent menacés (CHARAUDEAU, p. 125).

explicite de son ethos prédiscursif de féministe est son refus de tenir le rang de première dame aux bras de son époux lors de l'investiture de celui-ci. En effet, le 25 mai 2003, lors de l'investiture présidentielle du candidat du Frente Para la Victoria, Néstor Kirchner, son épouse, Cristina Fernandez de Kirchner, ne se tient pas à ses côtés mais occupe son siège de sénatrice dans l'hémicycle. Le titre de première dame n'étant qu'honorifique, elle lui préfère alors celui de femme politique active et se prédispose déjà, ainsi, à l'exercice de charges politiques plus importantes. 
Le DI est l'un des cadres les plus aboutis pour l'expression de cet ethos de solidarité. II apparait alors comme construisant du sujet politique une image d'homme appartenant à un groupe auquel il s'identifie et duquel il est à l'écoute dans le souci d'améliorer les conditions de vie de ses semblables. Le DI présidentiel, pour ce qui est de sa fonction programmatique annonçant une gestion meilleure et bénéfique de la nation, apparait alors comme étant de ces "formules qui ne garantissent aucunement qu'il y ait eu consultation du peuple mais qui disent "Je vous ai entendu" (CHARAUDEAU, p. 128). C'est tout le sens des promesses de bonheur et d'amélioration du cadre de vie qui ponctuent le DI. Ce sentiment de solidarité est stéréotypiquement exacerbé chez le genre féminin car si la doxa le lui attribue bien plus qu'à l'homme, les présidentes ci le revendiquent dans le cadre du DI. En témoignent ces extraits ${ }^{17}$ :

Je veux le dire à tous, en l'honneur des quarante millions d'Argentins (...) que nous allons continuer à travailler avec tous et pour tous pour une Argentine plus juste, plus équitable et plus solidaire.

Nous avons l'obligation à partir de l'Exécutif, du Parlement, de la Cour Suprême elle-même et des Tribunaux, d'adopter et de concevoir les instruments qui assurent tous les droits et garanties que d'autres argentins n'ont pas eu et enfin permettent de poursuivre et punir ceux qui auront été responsables du plus grand génocide de notre Histoire. Nous le devons à ceux qui en ont été les victimes; Nous le devons à leurs proches, aux Grand-mères, aux Mères, nous le devons aux survivants qui ne peuvent continuer à être soumis à la torture de l'histoire permanente de la tragédie.

(Cristina Fernández De Kirchner)

Christina Kirchner propose un long exposé sur la nécessité de la justice comme condition première de l'épanouissement de son peuple. Equité et solidarité sont des thèmes majeurs de son discours comme en témoigne la gradation ascendante qui clôt l'extrait initial. En promettant de travailler d'arrache pied pour une Argentine "plus juste, plus équitable, plus solidaire", la présidente investie se montre consciente des frustrations liées d'une part à l'incapacité pour les pauvres d'avoir accès à la justice et d'autre part à un partage biaisé des ressources économiques. Cette fracture sociale ne saurait perdurer si la solidarité animait chaque citoyen d'où l'appel implicite à la

\footnotetext{
${ }^{17}$ Nous soulignons.
} 
solidarité qui est ici réalisé. Mais l'énumération des victimes du génocide révèle non seulement que les femmes sont une frange marginalisée subissant avec plus d'acuité l'injustice sociale mais aussi que l'énonciatrice est particulièrement sensible à leur cause.

L'ethos de solidarité est manifeste chez Dilma Rousseff dans ce passage:

Je vous ai dit au début de ce discours, que je gouvernerai pour tous les Brésiliens et toutes les Brésiliennes. Et je vais le faire.

Le Brésil de l'avenir sera exactement à la mesure de ce que nous ferons pour lui aujourd'hui ensemble. À la mesure de la participation de tous et de chacun: des mouvements sociaux, de ceux qui labourent la terre, des professionnels libéraux, des ouvriers et des petits entrepreneurs, des intellectuels, des fonctionnaires, des entrepreneurs, des femmes, des noirs, des indiens et des jeunes, de tous ceux qui luttent pour surmonter les différentes formes de discrimination.

Je veux être aux côtés de ceux qui travaillent pour le bien du Brésil dans la solitude amazonienne, dans la sécheresse du Nord-Est, dans l'immensité du cerrado, dans la vaste étendue de la pampa.

Je veux être aux côtés de ceux qui vivent dans les agglomérations métropolitaines, dans l'immensité des forêts, dans l'arrière-pays ou sur la côte, dans les capitales des états et sur les frontières du Brésil.

(Dilma Rousseff)

Ce passage est initié par une parole de promesse (Je veux être aux côtés...), consistant à énoncer la volonté de la locutrice de gouverner sans discrimination. Toutefois la présidente investie introduit une restriction liée à l'incapacité des gouvernants à tenir de tels engagements sans la solidarité du peuple non seulement envers la classe dirigeante mais aussi et surtout envers la nation toute entière. Elle introduit donc une bipartition du concept de solidarité car celui-ci doit s'affirmer dans la verticalité (envers les gouvernants) et, conjointement, dans l'horizontalité (envers chaque citoyen de la nation). D'où la longue énumération des composantes du peuple à l'intention desquelles la citation tient aussi lieu d'appel, certes phatique, mais aussi d'exhortation intimant à chaque citoyen de sentir, à la fois que son sort est digne d'intérêt pour la présidente et qu'il se doit d'être solidaire de toute la nation. S'ensuit un argument par la volonté d'agir qui témoigne littéralement de l'ambition de la présidente investie d'être solidaire de son peuple, présent à 
ses côtés et continuellement disposé à lui apporter le soutien dont il aurait besoin pour s'épanouir.

Il en va de même pour Ellen Johnson Sirleaf chez qui la parole de promesse est exacerbée par l'usage d'un promissif explicite dans ce passage ${ }^{18}$ :

Comme vous le savez, dans nos différentes communautés et villes, nos enfants ont une façon propre d'accueillir leurs pères quand ils rentrent après une longue et fatigante journée à essayer de trouver les moyens de nourrir la famille chaque soir et d'envoyer les enfants à l'école le jour d'après. Ils disent: "Papa est venu!"

Eh bien, trop souvent, pour un trop grand nombre de familles, Papa rentre à la maison sans rien, n'ayant pas réussi à trouver un emploi ou à obtenir de l'aide pour nourrir les enfants affamés. Imaginez ensuite la déception et la souffrance de la mère et des enfants; la frustration et la perte de confiance en soi chez le père.

À travers le message de cette histoire, je veux que vous sachiez que je comprends ce que vous, nos citoyens ordinaires, traversez chaque jour pour joindre les deux bouts pour vous et pour vos familles. Les temps étaient durs avant. Les temps sont encore plus durs aujourd'hui. Mais je vous fais cette promesse: sous mon administration, nous travaillerons pour changer cette situation. Nous ferons en sorte que, lorsque nos enfants diront: "Papa est venu!", papa rentre joyeusement avec quelque chose, aussi maigre soit-il, pour subvenir aux besoins de sa famille.

(Ellen Johnson Sirleaf)

Le prétexte de la scène domestique contée est, comme le reconnait l'énonciatrice, de témoigner de sa compassion et de sa solidarité. La présidente investie se définit donc comme une composante du peuple. Elle en est du moins suffisamment proche pour savoir le quotidien de chaque enfant désœuvré et de chaque père de famille affligé de son impuissance quant à satisfaire les besoins essentiels de sa famille. L'épisode qu'elle décrit est d'un pathétique qui semble connoter la sincérité et la gravité. Toutes deux conférant à son promissif une réussite de rare portée perlocutoire du moment que sont simultanément convoqués l'ethos de solidarité et le pathos comme indice de rassemblement autour de valeurs communes bafouées par la précarité.

\footnotetext{
${ }^{18}$ Nous soulignons.
} 


\section{Conclusion}

Le DI est un genre normé et dont la codification laisse peu de place à des variations stylistiques et thématiques tranchées. Perçu d'un point de vue macro-structurel comme une parole de promesse du rassemblement autour de l'identité collective et des valeurs qui y sont attachées, les enjeux de la performativité qu'il actualise sont indissociables de l'aspiration à la légitimité qui en est au fondement. II reste cependant un lieu d'expression privilégiée de la revendication de l'identité féminine chez les présidentes concernées par cette étude. Ainsi outre l'argument du genre, les ethos d'humanité et de solidarité s'amplifient de la posture féministe des énonciatrices. Ceci pour la simple raison qu'ils actualisent le mythe de la femme-mère, de la mère nourricière, gardienne du foyer et de la stabilité de la cellule familiale qui sont autant de représentations sociales persistantes aussi bien en Afrique noire qu'en Amérique latine. Est-il besoin de rappeler que l'ethos est une image projetée et que par définition il ne peut prospérer sans se situer par rapport au sens commun? Si la doxa disqualifie les femmes quand il est question de pouvoir, revendiquer son genre vis-à-vis de telles représentations sociales dégradantes peut tenir lieu de nécessité. Les extraits présentés confirment cependant que de tels préjugés doxiques, s'ils constituent un handicap à l'émancipation féminine, sont déconstruits car réorientés axiologiquement, par les présidentes que nous avons citées, comme valeurs nobles et véritables. De ce fait si les hommes doivent en user avec parcimonie pour ne pas sembler efféminés, les femmes n'ont point à se priver de tels ethos assumant leur identité féminine et peuvent même en faire un usage abondant sans risquer de choquer l'opinion. D'où une forte propension à de tels ethos dans les DI féminins du corpus étudié. On aura donc distingué les "ethos de féminité" d'où la locutrice assume d'un point de vue subversif le rôle traditionnellement dévolu aux femmes par une réorientation axiologique - des "ethos féministes" qui prônent une performativité sociale du sexe, qui ne nécessite pas qu'une femme se définisse comme telle si son apparence le confirme déjà. Pour essentialiste que puisse sembler la perspective choisie, il s'est surtout agi de montrer que les "ethos de féminité" relèvent d'une perception dont les populations des pays concernés par cette étude sont coutumières en tant qu'impensé doxique et stéréotypique, et que les présidentes investies ne se 
positionnent que par rapport à cette représentation sociale de leur auditoire effectif.

\section{Références bibliographiques}

AEBISCHER, Verona. Les Femmes et le langage. Représentations sociales d'une différence. Paris: PUF, 1985.

AMOSSY, Ruth. La présentation de soi. Ethos et identité verbale. Paris: PUF, 2010.

AMOSSY, Ruth. L’argumentation dans le discours. Paris: Nathan Université, 2000.

BADINTER, Elisabeth. Le conflit: la femme et la mère. Paris: Flammarion, 2010

BENOIT À LA GUILLAUME, Luc. Les Discours d'investiture des présidents américains ou les paradoxes de l'éloge. Paris: l'Harmattan, 2000.

BOURDIEU, Pierre. La domination masculine. Paris: Seuil, 1998.

CHARAUDEAU, Patrick. Le discours politique: Les masques du pouvoir. Paris: Vuibert, 2005.

COULOMB-GULLY, Marlène. "Mâle ou normal?". Incarnation et masculinité(s) du couple Hollande-Sarkozy dans la campagne présidentielle de 2012. Genre, sexualité \& société, Hors-série, n. 2, 2013. DOI: 10.4000/gss.2619.

DERVILLE, Grégory; PIONCHON, Sylvie, La femme invisible. Sur l'imaginaire du pouvoir politique. Mots, n. 78, 2005. DOI: 10.4000/mots.369.

DUMONT, Louis. Homo Hierarchicus. 2. éd. Paris: Gallimard, 1979.

FERENCUHOVA, Maria. L'acte-lui, la parole-elle: Les personnages masculins et féminins chez Eric Rohmer. Revue Web Internationale, http://www.senspublic.org/article.php3?id_article=14.

HERITIER, Françoise. Masculin, Féminin. La pensée de la différence. Paris: Odile Jacob, 1996.

HRDY BLAFFER, Sarah. Les instincts maternels. Paris: Payot, 2002.

JAMI, Irène. Judith Butler, théoricienne du genre. Cahiers du Genre, n.44, p. 205-228, 2008.

LAMIZET, Bernard. Politique et identité. Lyon: Presses Universitaires Lyon, 2002. 
OGER, Claire. L'évaluation des campagnes dans le discours journalistique. Compétence attribuée et genre des candidats. Mots, n. 90, 2009. DOI: $10.4000 /$ mots. 19078

OGER, Claire. Du "parler cru" à l'insulte: niveaux de violence dans le discours sexiste en politique. In: MOÏSE Claudine; AUGER Nathalie; FRACCHIOLLA Béatrice; SCHULTZROMAIN, Christina (Éd.). La violence verbale. Espaces politiques et médiatiques. Paris: L'Harmattan, 2008.

OGER, Claire. Judith Butler, Le pouvoir des mots. Politique du performatif. Mots, n. 81, 2006. URL: http://mots.revues.org/736.

OLIVESI, Aurélie. L'interrogation sur la compétence politique en 2007: une question de genre?. Quaderni, n. 72, 2010. URL: http:// quaderni.revues.org/486.

PERELMAN, Chaïm; OLBRECHTS-TYTECA, Lucie. Traité de l'Argumentation. La nouvelle rhétorique. 6. éd. Bruxelles: Editions de l'Université de Bruxelles, 2008.

ROCHEBLAVE-SPENLE, Anne-Marie. Les rôles masculins et féminins: les stéréotypes, la famille, les états intersexuels. Paris: Presses Universitaires de France, 1964.

\section{Forma de citação sugerida}

Houessou, Dorgelès. Distinctions genrées et identités discursives dans la généricité du discours d'investiture: le cas de Kirchner, Rousseff et Sirleaf. EID\&A - Revista Eletrônica de Estudos Integrados em Discurso e Argumentação, Ilhéus, n. 19, p. 3-27, ago.2019. DOI dx.doi.org/10.17648/eidea-19-2301. 